\title{
Wireless Video Capsule Enteroscopy in Preclinical Studies: Methodical Design of Its Applicability in Experimental Pigs
}

\author{
Marcela Kopáčová · Ilja Tachecí · Jaroslav Květina • \\ Jan Bureš • Martin Kuneš • Stanislav Špelda • \\ Věra Tyčová $\cdot$ Zbyněk Svoboda $\cdot$ Stanislav Rejchrt
}

Received: 12 October 2008/Accepted: 23 February 2009/Published online: 18 March 2009

(c) The Author(s) 2009. This article is published with open access at Springerlink.com

\begin{abstract}
The aim of this project was to develop a methodology to introduce wireless video capsule endoscopy in preclinical research. Five mature female pigs (Sus scrofa domestica) were selected for the study. Capsule endoscopes (the EndoCapsule system; Olympus) were introduced into the duodenum endoscopically in each of the animals. The life span of batteries (i.e., total time of endoscopy recording) was 487-540 min (median $492 \mathrm{~min}$ ). The capsule endoscope reached the cecum during enteroscopy once (after $7 \mathrm{~h}$ $57 \mathrm{~min}$ ), in the remaining cases, endoscopy recordings terminated in the distal or terminal ileum. All capsule enteroscopies found a normal pattern of the small intestine. The intestinal lumen is narrower, transverse folds are sparse or even absent, villi are wider but less prominent in pigs
\end{abstract}

M. Kopáčová $(\bowtie) \cdot$ I. Tachecí · J. Bureš · S. Rejchrt Second Department of Internal Medicine, Faculty of Medicine at Hradec Králové, Charles University in Praha, University

Teaching Hospital, Sokolská 581, 500-05 Hradec Králové,

Czech Republic

e-mail: kopacmar@fnhk.cz

J. Květina $\cdot$ M. Kuneš $\cdot$ Z. Svoboda

Institute of Experimental Biopharmaceutics, Joint Research

Center of Czech Academy of Sciences and PRO.MED.CS Praha

a.s., Hradec Králové, Czech Republic

S. Špelda

Department of Radiobiology, Faculty of Military Health

Sciences, University of Defence, Hradec Králové,

Czech Republic

V. Tyčová

The Fingerland Department of Pathology, Faculty of Medicine at Hradec Králové, Charles University in Praha, University

Teaching Hospital, Hradec Králové, Czech Republic compared to humans. Capsule endoscopy in experimental pigs will be helpful for future trials on injury of different drugs and xenobiotics to the small bowel.

Keywords Capsule endoscopy · Enteroscopy · Experimental pigs $\cdot$ Small bowel

\section{Introduction}

Wireless video capsule endoscopy represents fundamental progress in non-invasive imaging of the gastrointestinal tract, particularly the small bowel. The method was introduced into clinical practice in 2001 and more than 500,000 examinations have been performed in humans worldwide up to the present time [1-5]. At a very early stage, an initial study on experimental use of capsule endoscopy in dogs was published [6]. However, other papers dealing with the use of capsule endoscopy in a different experimental setting are still missing. The aim of this project was to develop a methodology to introduce wireless video capsule endoscopy in experimental pigs. The small adult pig can be used in experiments as an omnivorous representative due to its relatively very similar gastrointestinal functions in comparison to humans [7].

\section{Animals and Methods \\ Preparation for the Project}

In a pilot test on experimental pigs, we proved that endoscopic insertion of video capsules into the duodenum is feasible. A transverse semi ball-shaped pyloric fold (torus 
pyloricus) was found in the porcine stomach of all animals, serving as a "gatekeeper" and thus making the endoscopic insertion technically difficult. The position of capsule endoscopes was set in three animals: in the proximal jejunum $(330 \mathrm{~cm}$ ) after $90 \mathrm{~min}$, in the proximal ileum after $180 \mathrm{~min}$, and in the cecum after $9 \mathrm{~h}$. In another four animals, the time until natural excretion of capsule endoscopes in the feces was measured, this was $72,48,40$, and $65 \mathrm{~h}$ (mean $56 \pm 15 \mathrm{~h}$ ). The length of the small intestine of 4-monthold pigs was found to be highly variable. This was measured at immediate autopsy in 13 animals, ranging from 11.2 to $16.8 \mathrm{~m}$ (mean $13.4 \pm 1.8 \mathrm{~m}$ ).

\section{Methodical Design}

Five mature female pigs (Sus scrofa f. domestica), hybrids of Czech White and Landrace breeds, weighing $36.6 \pm$ $3.0 \mathrm{~kg}$ (4-5 months old) were entered into the study. An EndoCapsule system (Olympus Optical Co, Tokyo, Japan) was used for video capsule enteroscopies in all animals. The wireless capsule endoscope has an outer diameter of $11 \mathrm{~mm}$, length of $26 \mathrm{~mm}$, and weighing $3.8 \mathrm{~g}$. Capsule endoscopes were introduced into the duodenum endoscopically in all animals. Endoscopy procedures were performed using video-gastroscopes GIF-Q130 (Olympus Optical Co, Tokyo, Japan) dedicated for animal use only. All endoscopies were recorded on DVD. Four capsule endoscopes were placed into the duodenum by means of a G25 retrieval basket (Sun, Vikýřovice, Czech Republic) and the remaining one using the AdvanCE delivery system (US Endoscopy, Mentor, USA).

Capsule enteroscopies were carried out in the stable phase of general anesthesia (intramuscular bolus of $20 \mathrm{mg} / \mathrm{kg}$ ketamine "Narkamon-Spofa, Praha, Czech Republic" $+2 \mathrm{mg} / \mathrm{kg}$ azaperone "Stresnil—Jansen Pharmaceutica, Beerse, Belgium") on supine position in all animals (Fig. 1). Repeated doses of $1 \%$ thiopental (Thiopental, Valeant Czech Pharma, Prague, Czech Republic) were administrated when appropriate to the lateral auricle vein. Syntostigmine (0.5 mg i.v.-Hoechst-Biotika, Martin, Slovakia) was administrated immediately after successful placement of the capsule endoscope into the duodenum (to eliminate the influence of general anesthesia on the gastrointestinal passage). Infusions of $0.9 \%$ saline solution were chosen to secure basal hydration $(1,000 \mathrm{ml} / 8 \mathrm{~h})$. All animals were covered with blankets to prevent hypothermia during capsule enteroscopy. The procedure was finished after discharge of the batteries.

Data obtained during capsule enteroscopy were downloaded from the recorder unit to the workstation. It contains proprietary application software "Endo Capsule Software" (Olympus Optical Co, Tokyo, Japan), which enables the smooth management of capsule endoscope examination

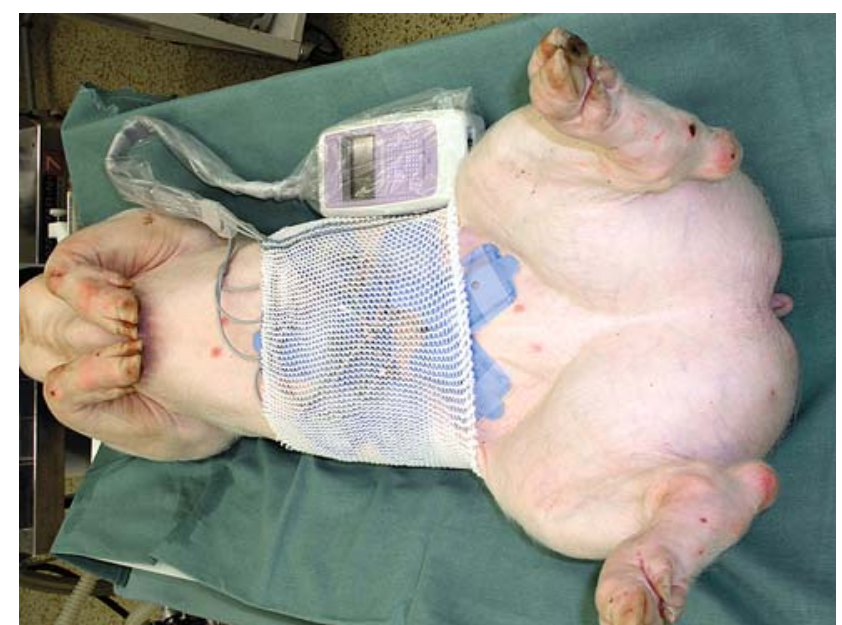

Fig. 1 Capsule endoscopy in an experimental pig. General view of the arrangement of investigation. The antenna system is affixed to skin of the abdomen; the data recorder is next to it

data. All video images were evaluated by a single physician, using usually auto speed adjustment (the review speed is automatically increased when there is little movement in consecutive images) with the lower limit for 12 -frames/s of review speed in most recordings.

The next morning the pigs were sacrificed by means of pharmacological euthanasia (T61, Intervet International $\mathrm{BV}$, Boxmeer, the Netherlands; dose of $2 \mathrm{ml} / \mathrm{kg}$ ). Immediate autopsy was performed and samples collected of the pyloric transverse fold, and specimens of gastric and intestinal segments for light microscopy.

\section{Ethics}

The project was approved by the Institutional Review Board of the Animal Care Committee at the Institute of Experimental Biopharmaceutics, Academy of Sciences of the Czech Republic, Protocol Number 149/2006. Animals were held and treated in accordance with the European Convention for the Protection of Vertebrate Animals Used for Experimental and Other Scientific Purposes [8].

\section{Results}

Capsule endoscopy was successful in all animals. Endoscopical placement of the capsule endoscope into the duodenum took 1.5 to $8 \mathrm{~min}$ (mean $5.5 \mathrm{~min}$, median 6 min) using a basket and 42 min using a special delivery system. Endoscopic introduction of the capsule endoscope into the duodenum was difficult because of the pyloric transverse fold (see Fig. 2) that makes the pylorus relatively stenotic for endoscopic accessories. This fold (called 


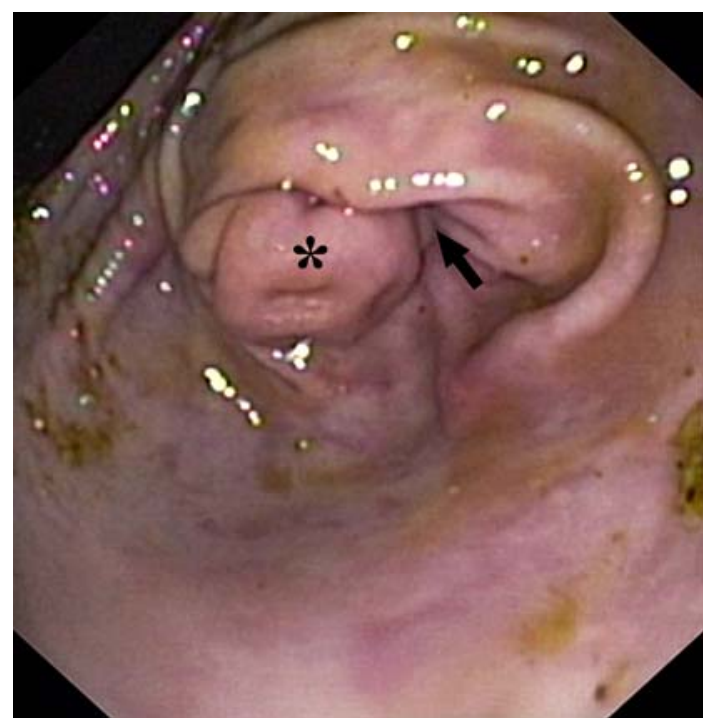

Fig. 2 Endoscopic view of the transverse pyloric fold-torus pyloricus (asterisk) in a porcine stomach. This fold makes the pylorus (arrow) relatively stenotic for endoscopic accessories

"torus pyloricus") is constituted by the ovoid-shaped muscularis propria and enlarged mucosa (Fig. 3).

The life span of batteries (i.e., total time of the endoscopy recording) was 487-540 min (mean $505 \pm 22 \mathrm{~min}$, median $492 \mathrm{~min}$ ). The capsule endoscope reached the cecum during enteroscopy once (at $7 \mathrm{~h} 57 \mathrm{~min}$ ), in the remaining cases, endoscopy recordings terminated in the distal or terminal

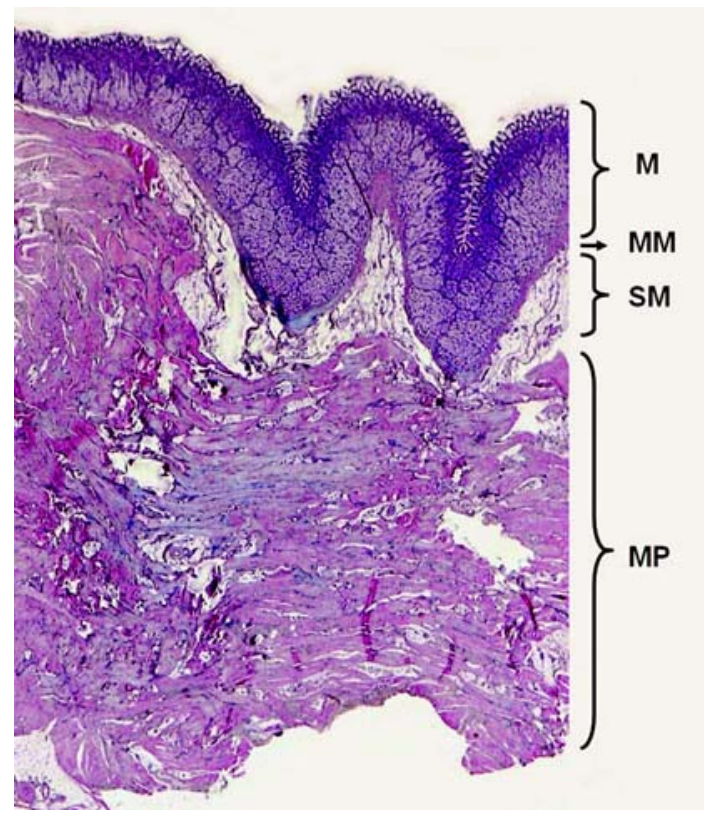

Fig. 3 Histology of the transverse pyloric fold in a porcine stomach. This fold is created mostly by the ovoid-shaped capacious muscularis propria (MP) and enlarged mucosa (M). The normal submucosa is marked as SM, the muscularis mucosa is lettered as MM. Hematoxylin-eosin, magnification $10 \times$ ileum. Upon autopsy the next day after capsule enteroscopy, the capsule endoscopes were found in the cecum in all cases.

Total length of the entire small intestine, including the duodenum (at immediate autopsy) was 11.2-13.7 m (mean $12.2 \pm 1.1$; median $11.7 \mathrm{~m}$ ). It was not possible to set the exact borderline between the jejunum and ileum either endoscopically (during capsule enteroscopy), or macroscopically (at autopsy) or histologically. Approximately half comprises the jejunum and the other half the ileum. Movement of the capsule endoscope had less regional transit abnormalities in the duodenum and jejunum as compared to the ileum. In our pilot testing in one animal before this trial, the mean speed of the capsule endoscope in the proximal and middle jejunum was $4 \mathrm{~cm} / \mathrm{s}$. In the present study, there were several periods of persistence of capsule endoscope at one location without any propulsive movement (5-60 min). A rough estimation of the approximate location of the capsule endoscope (after its introduction into the duodenum) at the time of recording was as follows: the proximal jejunum at $10 \mathrm{~min}$, middle jejunum at $60 \mathrm{~min}$, borderline of the jejunum and ileum at approximately $150 \mathrm{~min}$, middle ileum at $270 \mathrm{~min}$, distal ileum at $450 \mathrm{~min}$, terminal ileum at $>480 \mathrm{~min}$. In case of persistence of the capsule endoscope in one place without any propulsive movement, this time is subtracted (from the time line) to estimate the approximate position of the capsule endoscope.

The quality of the small-intestinal images ranged from very good to excellent in the jejunum in all animals (Fig. 4). Due to the large volume of intestinal content in the ileum, the visibility of some ileal segments was a little bit worse. There were no technical complications (e.g., early discharge of the battery, loss of data recording etc.). All capsule enteroscopies found a normal pattern of the small intestine in all experimental animals. A larger volume of intestinal juice with a marked content of bile was constantly seen in the small intestinal lumen in all enteroscopies. The endoscopic pattern of the small bowel in experimental pigs seems to be slightly different from that seen in humans (compare Figs. 4, 5). The intestinal lumen is narrower, transverse folds are sparse or even absent, villi are wider but less prominent in experimental pigs compared to humans.

\section{Discussion}

The aim of this paper was to work out a methodology and to set up capsule enteroscopy in experimental pigs (as an omnivorous representative metabolically close to humans). Our study produced new data on capsule endoscopy in experimental pigs. Capsule enteroscopy was accomplished 


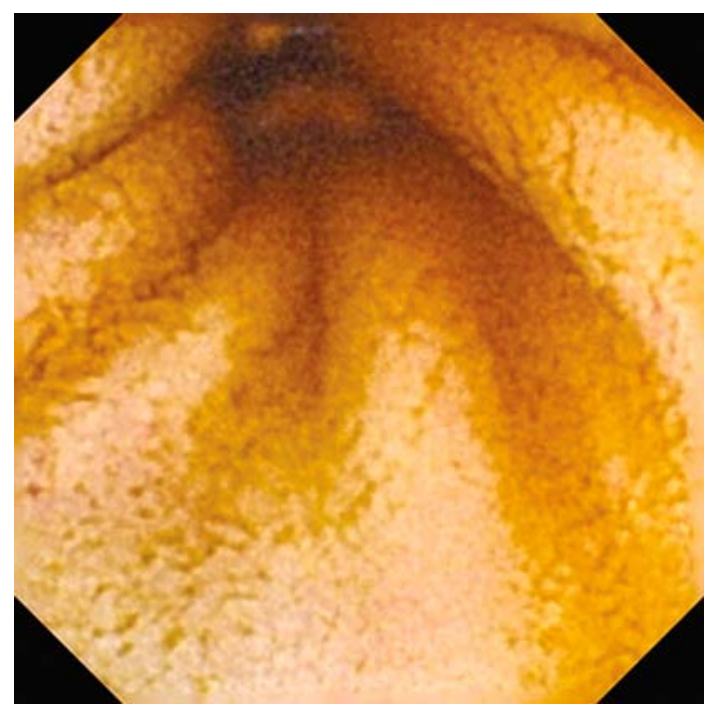

Fig. 4 Enteroscopy picture of the normal porcine jejunum (capsule endoscopy by means of Olympus EndoCapsule System). Transverse folds are absent, villi are wider but less prominent. A large volume of intestinal juice with a marked content of bile is seen

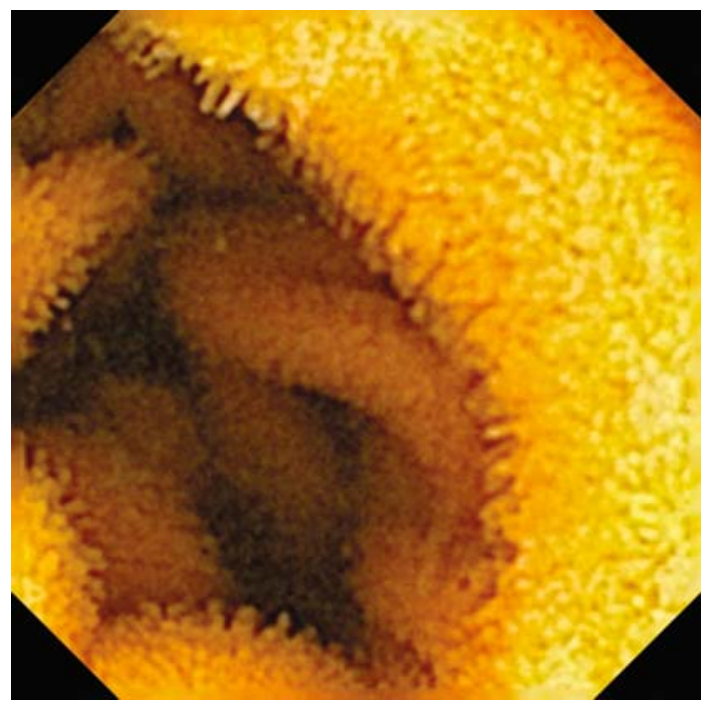

Fig. 5 Immersion enteroscopy picture of the normal human jejunum (capsule endoscopy by means of EndoCapsule System Olympus). Transverse folds (plicae circulares Kerkringi) are regular and dense, villi are clearly visible, being high and spiky. The intestinal lumen is usually empty. Picture reprinted from Tachecí et al. [10], reproduced with permission

in all animals. Thus, this method is feasible in an experimental setting in pigs. However, it was necessary to overcome several difficulties. Flexible upper endoscopy is more demanding in pigs (compared to humans). The mean distance from incisors to the gastro-esophageal junction is about $60 \mathrm{~cm}$ in these pigs. The porcine stomach is pouchshaped and gastric cardia is close to the pylorus, so the endoscopic approach to the duodenum is rather hooked. To prevent a delay due to persistence of the video capsule in the stomach, we introduced all capsules directly into the duodenum by means of a standard flexible video-gastroscope. Pigs have an epipharyngeal diverticulum so it was necessary to introduce the gastroscope with a grasped capsule very carefully so as not to lose it during insertion and to prevent aspiration of the capsule. There was a distinct difference in the time necessary for gastroscopic introduction of capsule into the duodenum, using a basket and special delivery system. There is a transverse pyloric fold (torus pyloricus) in the porcine stomach that makes the pylorus relatively stenotic for endoscopic accessories. The outer diameter of the special delivery system is $13 \mathrm{~mm}$ and it must be introduced in a prograde way. We succeeded only after several repeated attempts using this system. The capsule grasped into a basket was introduced sideways and proved much easier and shorter (outer diameter of the capsule is $11 \mathrm{~mm}$ ).

The entire small intestine of a young adult pig (weighing $35 \mathrm{~kg}$ ) measures about $12 \mathrm{~m}$ in length immediately after pharmacological euthanasia. This is twice as long as in an adult human (weighting $70 \mathrm{~kg}$ ) despite the fact that the pig is an omnivore like humans. The movement of capsule endoscope was more rapid in the jejunum compared to the ileum. This fact reflects the natural motility character of the jejunum. Furthermore, syntostigmine was administrated at the beginning of the experiment to enhance the propulsive work of the small intestine and to forestall any negative effect of general anesthesia on gastrointestinal motility. Half-time of syntostigmine is only $1-2 \mathrm{~h}$ so it cannot assure stimulated motility during the whole investigation. IV infusions to secure proper hydration and covering animals with blankets to forestall hypothermia during capsule enteroscopy are mandatory to prevent further negative effects on the small intestinal motility.

The mean life span of the batteries used in the experiment was about $500 \mathrm{~min}$. Capsule enteroscopy (similar to other methods) is not able to recognize the exact borderline between the jejunum and ileum. We have proposed a rough estimation of the approximate location of the capsule endoscope, being merely a conservative estimate. We are fully aware of the limits of this proposal (inter-individual variability in the motility pattern, absence of reliable landmarks, the cecum was reached only in one animal during capsule enteroscopy recording etc.). In clinical studies in humans (having the small bowel twice shorter than a pig), the cecum is reached; that means the entire small intestine is investigated in only about $75 \%$. In up to $25 \%$ of cases, the batteries also run out before the capsule passes the ileo-cecal valve in humans [9].

In summary, wireless video capsule endoscopy is feasible in experimental pigs. This paper concerns methodological 
preparation for further experimental studies on (1) the effect of different drugs and xenobiotics on the small intestine and (2) the mechanism of pharmacokinetics of different pharmaceutical formulations of drugs in the small bowel. Our study provided several new data, differentiating capsule endoscopy in experimental pigs from those performed in humans.

Acknowledgments The endoscopic part of the study was supported by research project MZO 00179906 from the Ministry of Health, Czech Republic. The experimental part of the study was supported by research grant GAČR 305/08/0535, Czech Republic.

Open Access This article is distributed under the terms of the Creative Commons Attribution Noncommercial License which permits any noncommercial use, distribution, and reproduction in any medium, provided the original author(s) and source are credited.

\section{References}

1. Eliakim R. Video capsule endoscopy of the small bowel. Curr Opin Gastroenterol. 2008;24:159-163. doi:10.1097/MOG.0b013 e3282f3d946.
2. El-Matary W. Wireless capsule endoscopy: indications, limitations, and future challenges. J Pediatr Gastroenterol Nutr. 2008; 46:4-12. doi:10.1097/01.mpg.0000313818.96520.6e.

3. Keuchel M, Hagenmüller F, Fleischer DE, eds. Atlas of video capsule endoscopy. Heidelberg: Springer; 2006.

4. Mishkin DS, Chuttani R, Croffie J, et al. ASGE technology status evaluation report: wireless capsule endoscopy. Gastrointest Endosc. 2006;63:539-545. doi:10.1016/j.gie.2006.01.014.

5. Sidhu R, Sanders DS, Morris AJ, McAlindon ME. Guidelines on small bowel enteroscopy and capsule endoscopy in adults. Gut. 2008;57:125-136. doi:10.1136/gut.2007.129999.

6. Appleyard M, Fireman Z, Glukhovsky A, et al. A randomized trial comparing wireless capsule endoscopy with push enteroscopy for the detection of small-bowel lesions. Gastroenterology. 2000;119:1431-1438. doi:10.1053/gast.2000.20844.

7. Kararli TT. Comparison of the gastrointestinal anatomy, physiology and biochemistry of humans and commonly used laboratory animals. Biopharm Drug Dispos. 1995;16:351-380. doi:10.1002/ bdd. 2510160502.

8. Explanatory report on the European convention for the protection of vertebrate animals used for experimental and other scientific purposes. Strasbourg: Council of Europe; 1986.

9. Cave D. Wireless video capsule endoscopy. Available from www.uptodate.com. 2008; vol. 16.2. Accessed September 20, 2008.

10. Tachecí I, ed. Capsule endoscopy. Hradec Králové: Nucleus HK; 2008. 